\title{
Serious complications following treatment of chronic idiopathic thrombocytopenic purpura
}

\author{
W. Wanachiwanawin, S. Visudhiphan, A. Piankijagum and S. Vatanavicharn \\ Department of Medicine, Faculty of Medicine, Siriraj Hospital, Mahidol University, Bangkok 10700, Thailand.
}

\begin{abstract}
Summary: Six patients had serious complications as consequences of treatment of idiopathic thrombocytopenic purpura. Five had splenectomy-related complications, one of them developed fatal intra-abdominal bleeding. Three patients acquired operation-related serious infection, two of them died. Serious neutropenia after vinblastine-loaded platelets occurred in one patient leading to pseudomonas septicaemia and panophthalmitis with permanent vision loss of left eye. Recurrence thrombocytopenia occurred in every case during serious complications. Early detection by awareness of the possibility of serious complications can reduce morbidity and mortality occurring after therapy of idiopathic thrombocytopenic purpura.
\end{abstract}

\section{Introduction}

Chronic idiopathic thrombocytopenic purpura (chronic ITP) is an autoimmune disorder characterized by destruction of autoantibody-bound platelets by the reticuloendothelial system. ${ }^{1,2}$ In most cases, the responsible autoantibody is IgG with or without fixed complement $\mathrm{C} 3 .^{3}$

Various methods have been used for the treatment of chronic ITP. Splenectomy and administration of corticosteroids are the mainstays ${ }^{4,5}$ but approximately $25 \%$ of patients have disease refractory to these conventional measures. The patients in whom refractory ITP develops undergo therapy with vincristine, azathioprine, cyclophosphamide, vinblastine-loaded platelets, danazol, colchicine and high-dose intravenous gammaglobulin. 2,4,5,6 Each measure can lead to undesirable side effects, and since many patients receive more than one therapeutic regimen, serious consequences may occur. These complications, mostly infection related, can lead to serious, and sometimes fatal, damage especially if they are not recognized.

We report here serious complications following treatment of 6 chronic ITP patients.

\section{Case reports}

During January 1962 to December 1985, 698 ITP patients were seen at our haematology unit.

Correspondence: W. Wanachiwanawin, M.D.

Accepted: 22 December 1987
Diagnosis was made according to published criteria. $^{7}$ All cases received corticosteroids as initial therapy. Therapeutic splenectomies were performed in 146 patients $(20 \%), 43$ of whom had significant and prolonged episodes of thrombocytopenia after splenectomy and were considered to have refractory ITP. Some refractory cases received immunosuppressive agents alone or combined with corticosteroids as long-term or intermittent therapy. Six patients (detailed below) were found to have serious consequences of treatments. The relevant clinical data are summarized in Table I.

\section{Case 1}

A 21 year old woman was referred from another hospital after failure of conventional corticosteroid administration for ITP. When she was first seen in our haematology clinic, a severe cushingoid appearance was observed. Splenectomy was performed and resulted in the platelet count rising from 0 to $100 \times 10^{9} / 1$ in 5 days. On the seventh day postoperatively, she complained of back pain which was followed by confusion and a hypotensive episode. In spite of whole blood transfusion her condition deteriorated and sudden cardiac arrest developed before an exploratory laparotomy could be done. Autopsy revealed haemoperitoneum of 3 litres and also haemorrhage with necrosis of the tail of the pancreas.

Case 2

A 43 year old woman attended the Gynaecology

(C) The Fellowship of Postgraduate Medicine, 1988 


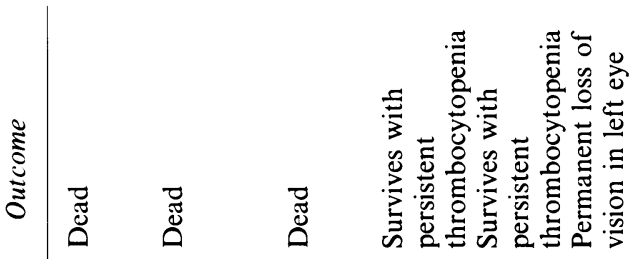

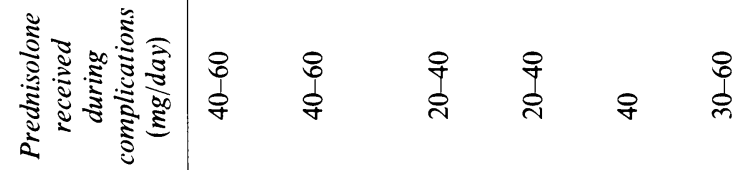

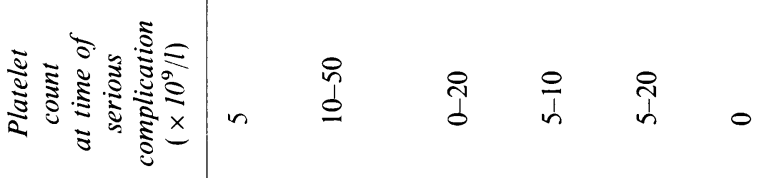

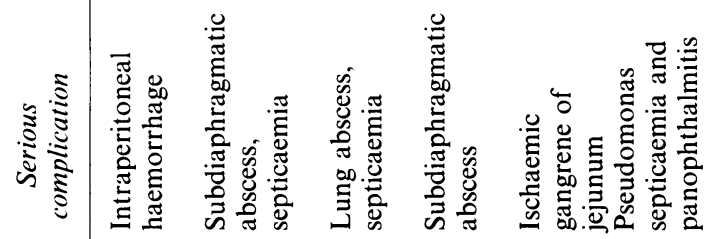

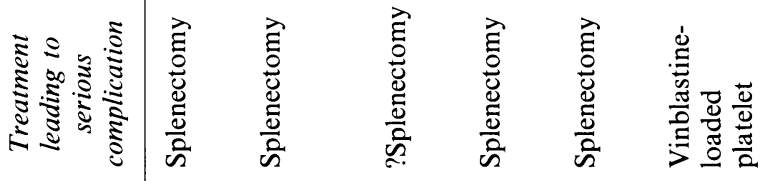

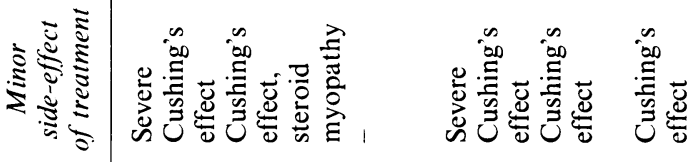

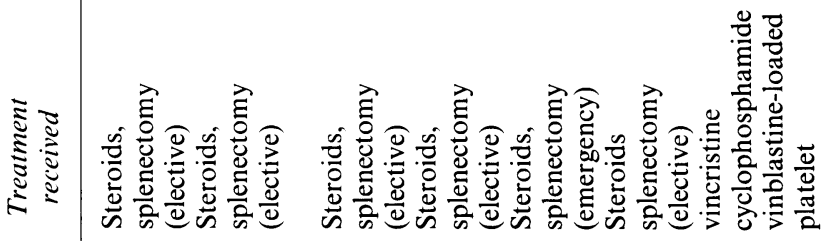

융

흘잉

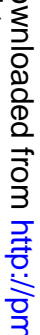

훙.

ญิ

옥

을.

స్

谂

ए)

잉

$\stackrel{\mathscr{\infty}}{\stackrel{9}{+}}$

꿍 
Department because of hypermenorrhea for 3-4 months. Dilatation and curettage showed endometrial hyperplasia with squamous metaplasia of the endometrium. Bleeding per vaginam continued after curettage and the complete blood count showed moderate thrombocytopenia. The bone marrow examination was consistent with ITP. Prednisolone 40 to $60 \mathrm{mg}$ per day resulted in a platelet count of $100 \times 10^{9} / 1$ within 4 weeks of medication. Bleeding per vaginam decreased but was not entirely stopped. After abdominal hysterectomy and splenectomy at the same time the platelet count rose to $130 \times 10^{9}-450 \times 10^{9} / 1$. Prednisolone was tapered off in 6 weeks, after which low grade fever occurred. She was readmitted to the hospital 4 weeks later. A provisional diagnosis of systemic lupus erythematosus was made on the basis of fever, alopecia, and polyarthralgia but the immunological profiles did not confirm this diagnosis. She was discharged 3 weeks later on prednisolone $60 \mathrm{mg} / \mathrm{day}$ but was admitted again 2 weeks later because of high grade fever. At this time, clinical examination led to a diagnosis of subdiaphragmatic abscess, confirmed by exploratory laparotomy. Her postoperative condition was complicated by enterocutaneous fistula and septicaemia which did not respond to systemic antibiotics. She expired 3 weeks after the last operation.

\section{Case 3}

A 40 year old woman was first seen 5 years previously because of anaemia and thrombocytopenia. Evaluation at that time pointed to the diagnosis of autoimmune haemolytic anaemia and thrombocytopenia (Evans' syndrome). Repeated studies of LE cells and antinuclear factors were negative. Prednisolone and cyclophosphamide were used with partial response and occasional relapse of anaemia and thrombocytopenia. One serious episode of thrombocytopenia required therapeutic splenectomy which brought the platelet count to normal level within one week postoperatively. Pneumonia of the left lower lobe and transient thrombocytopenia developed 4 weeks later. Conventional antibiotic therapy improved the infectious process and corticosteroids reversed thrombocytopenia to normal. However, 4 weeks later both episodes relapsed with progression of pneumonia to lung abscess. The responsible organism was identified as Proteus species and appropriate antibiotics were used. Mucosal bleeding required high dose corticosteroids which retarded clearance of infection. Uncontrollable sepsis was the leading cause of death 10 . weeks after last admission.

\section{Case 4}

A 20 year old woman was diagnosed as having ITP and referred from a rural hospital because of poor response to corticosteroids. Elective splenectomy was considered. The operation was uneventful but the postoperative condition was complicated by fever and recurrent thrombocytopenia on the ninth day postoperatively. Exploratory laparotomy revealed a left subdiaphragmatic collection from which bacteriological culture showed the same microorganism as on blood culture, i.e., Salmonella group $B$. It took at least 5 weeks after the last operation before she could be discharged from the hospital with continued thrombocytopenia.

\section{Case 5}

A 40 year old woman was first diagnosed as having ITP in 1963 when she was 17 years old. The major problems were generalized ecchymoses and hypermenorrhoea. Prednisolone was given intermittently during bleeding episodes with partial response. She refused therapeutic splenectomy. In January 1985, she experienced sudden onset of severe headache. Computed tomography showed an intracerebral haematoma at the left angular gyrus. The platelet count at that time was zero. Emergency splenectomy was performed and the platelet count rose to $20 \times 10^{9} / 1$ with improvement of the neurological deficits. She was maintained on prednisolone $40 \mathrm{mg} /$ day. Three weeks after splenectomy she experienced sudden onset of generalized abdominal pain. Physical examination revealed signs of peritoneal irritation. The platelet count remained below $20 \times 10^{9} / 1$. Exploratory laparotomy was decided on after 4 units of platelet concentrate were transfused. Serosanguinous ascites and a sixinch gangrenous segment of jejunum was found, 6 inches away from the duodeno-jejunal junction. Resection with end-to-end anastomosis was performed without postoperative complication. Surgical section revealed thrombotic occlusion of corresponding mesenteric veins.

\section{Case 6}

A 19 year old woman had petechiae and ecchymoses 3 months before ITP was diagnosed. Her thrombocytopenia was partially reversed by high dose prednisolone (more than $40 \mathrm{mg} /$ day). The Cushing effect was, however, intense and splenectomy was decided on. Complete recovery was achieved for only 3 months postoperatively and thrombocytopenia with occasional mucosal bleeding recurred. Prednisolone and cyclophosphamide were 
used without any promising result. Three daily doses of vinblastine-loaded platelets were tried. High fever, diarrhoea, and abdominal distension developed at the same time when marked leukopenia $\left(1.3 \times 10^{9} / 1\right)$ occurred, 2 days after the last dose of vinblastine-loaded platelets. A panophthalmitis rapidly followed which proved to be due to Ps. aeruginosa septicaemia. She subsequently recovered from the serious episode but with permanent loss of vision in her left eye.

A summary of clinical data is shown in Table I. All patients received corticosteroids as an initial treatment and were either steroid-nonresponsive or steroid-dependent. Cushing's effect developed in 5 patients, and was severe in two. Serious complications occurred as a consequence of splenectomy in 5 patients (Cases 1-5). One patient (Case 6) had pseudomonas septicaemia following vinblastineinduced leukopenia. Three who had splenectomyrelated complications died, one from massive intra-abdominal bleeding, two from uncontrollable infection. Thrombocytopenic episodes usually occurred during serious complications.

\section{Discussion}

Corticosteroids are accepted as conventional treatment of ITP. ${ }^{7}$ However, long-term corticosteroid administration can lead to many undesirable effects. Among them are hypertension, hyperglycaemia, Cushing's appearance, myopathy and increased susceptibility to infection. ${ }^{8}$ These side effects, especially the last one, may have some considerable influence on the outcome of splenectomy. Our first case demonstrated an effect of long-term corticosteroid administration on tissue healing. Rupture of a ligated vessel was a possible cause of the fatal intra-abdominal bleeding. Corticosteroids might have delayed clearance of bacterial infections in Cases 2-4.

Splenectomy has been accepted as an effective treatment of ITP after failure of corticosteroids. Serious complication following splenectomy is uncommon. ${ }^{9}$ Block et al. ${ }^{10}$ reported three postoperative deaths $(4.4 \%)$ among 67 ITP patients undergoing splenectomy. In their series two patients died within 10 days after operation from the effects of haemorrhage. It is possible that corticosteroids

\section{References}

1. McMillan, R. Chronic idiopathic thrombocytopenic purpura. N Engl J Med 1981, 304: 1135-1147.

2. Karpatkin, S. Autoimmune thrombocytopenic purpura. Semin Hematol 1985, 22: 260-288. might have retarded clearance of microorganisms leading to uncontrollable infections. Corticosteroids may also hamper inflammatory reaction and delay early recognition of infectious process, as occurred in patients 2 and 3.

One patient had ischaemic gangrene of the jejunum 3 weeks after splenectomy. This complication was not the result of rebound thrombocytosis since the platelet count was low at that time. Although detection of lupus circulating anticoagulant $^{11}$ was not done, the negative immune profiles and the occurrence of such complications at short duration after splenectomy made lupus circulating anticoagulant an unlikely cause of intestinal ischaemia. The relationship between splenectomy and intestinal gangrene is unknown although one can speculate about disturbance of the mesenteric circulation during the operation.

A variety of non-steroidal immunosuppressive agents have been used in the treatment of ITP refractory to splenectomy and use of corticosteroids. In order to reduce both short and longterm hazards from such agents, a variety of therapeutic options including dosage modification have been tried. Vinblastine-loaded platelets were claimed to be effective in refractory ITP. ${ }^{12}$ Unless removal of free drug is inadequate, serious side effects including neutropenia are unusual. Our patient 6 was an exceptional case, having severe granulocytopenia following vinblastine-loaded platelets. Permanent loss of vision in her left eye was a serious consequence of complicating pseudomonas septicaemia and panophthalmitis.

Clinicians need to be aware that serious complications may occur in ITP patients during treatment. Early recognition of such complications is the only way to save the patients. Among predisposing factors are combination therapy especially with corticosteroids and splenectomy. Elective splenectomy should be avoided in any patients with Cushing's effect. Recurrent thrombocytopenia was a constant finding and may be used, combined with other manifestations, as a warning sign of serious consequences.

\section{Acknowledgements}

We wish to thank all clinicians and surgeons who took care of the patients and Ms Charunee Tipdee for her secretarial help.

3. Burns, T.R. \& Saleem A. Idiopathic thrombocytopenic purpura. Am J Med 1983, 75: 1001-1007. 
4. Ahn, Y.S. \& Harrington, W.J. Treatment of idiopathic thrombocytopenic purpura. Ann Rev Med 1977, 28: 299-309.

5. Rosse, W.F. Treatment of chronic immune thrombocytopenia. Clin Haematol 1983, 12: 267-284.

6. Ahn, Y.S., Harrington, W.J., Simon, S.R. et al. Danazol for the treatment of idiopathic thrombocytopenic purpura. $N$ Engl J Med 1983, 308: 1396-1399.

7. Karpatkin, S. Autoimmune thrombocytopenic purpura. Blood 1980, 56: 329-343.

8. Haynes, R.C. Jr. \& Murad, F. Adrenocorticotropic hormone. In: Gilman, A.G., Goodman, L.S. \& Gilman, A. (eds). The Pharmacological Basis of Therapeutics, 6th ed. Macmillan, New York, 1980, pp. $1466-1496$
9. Difino, S.M., Lachant, N.A., Kirshner, J.J. \& Gottlieb, A.J. Adult idiopathic thrombocytopenic purpura: Clinical findings and responses to therapy. Am J Med 1980, 69: 430-442.

10. Block, G.E., Evans, R. \& Zajtchuk, R. Splenectomy for idiopathic thrombocytopenic purpura. Arch Surg 1966, 92: 484-489.

11. Sukenik, S., El-Roeiy, A. \& Shoenfeld, Y. Lupus anticoagulant and anticardiolipin antibodies in systemic lupus erythematosus. Acta Haematol 1986, 76: $86-89$.

12. Ahn, Y.S. The treatment of idiopathic thrombocytopenic purpura with vinblastine-loaded platelets. $N$ Engl J Med 1978, 298: 1101-1107. 